\title{
Alterations in fecal Lactobacillus and Bifidobacterium species in type 2 diabetic patients in Southern China population
}

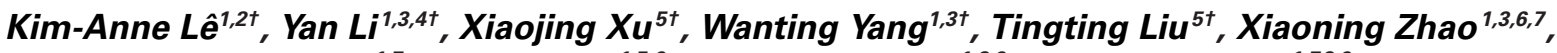 \\ Yongming Gorge Tang ${ }^{1,7}$, Dehong Cai ${ }^{1,5,6}$, Vay Liang W. Go ${ }^{1,8,9}$, Stephen Pandol ${ }^{1,7,8,9}$ and \\ Hongxiang Hui ${ }^{1,3,4,5,6,8,9 *}$
}

1 International Center for Metabolic Diseases, Southern Medical University, Guangzhou, China

${ }^{2}$ Nutrition and Health Department, Nestec Ltd., Nestlé Research Center, Lausanne, Switzerland

${ }^{3}$ Dongguan SMU Metabolic Medicine Inc. Ltd., Dongguang, China

${ }^{4}$ School Biotechnology, Southern Medical University, Guangzhou, China

${ }^{5}$ Department of Endocrinology, Zhujiang Hospital Southern Medical University, Guangzhou, China

${ }^{6}$ Center of Metabolic Diseases, Beijiao Hospital, Southern Medical University, Guangzhou, China

7 Department of Medicine, Cedars-Sinai Medical Center, Los Angeles, CA, USA

${ }^{8}$ Department of Medicine, VA Greater Los Angeles Health Care System, Los Angeles, CA, USA

${ }^{9}$ UCLA Center for Excellence in Pancreatic Diseases, David Geffen School of Medicine at University of California, Los Angeles, Los Angeles, CA, USA

\section{Edited by:}

Stephen O'Keefe, University of Pittsburgh Medical Center, USA

\section{Reviewed by:}

Patrick Tso, The University of Cincinnati Medical Center, USA

Christopher Chang, Cedars-Sinai Medical Center, USA

*Correspondence:

Hongxiang Hui, International Center for Metabolic Diseases,

Southern Medical University,

8 Floor, Life Science Build,

North 1838 Guangzhou Road,

Guangzhou 510515, China.

e-mail: huihongx@gmail.com

t These authors equally contributed to this work
Background: The connection between gut microbiota and metabolism and its role in the pathogenesis of diabetes are increasingly recognized. The objective of this study was to quantitatively measure Bifidobacterium and Lactobacillus species, members of commensal bacteria found in human gut, in type 2 diabetic patients (T2D) patients from Southern China.

Methods: Fifty patients with T2D and thirty control individuals of similar body mass index (BMI) were recruited from Southern China. T2D and control subjects were confirmed with both oral glucose tolerance test (OGTT) and $\mathrm{HbA}_{1 \mathrm{c}}$ measurements. Bifidobacterium and Lactobacillus species in feces were measured by real-time quantitative PCR. Data were analyzed with STATA 11.0 statistical software.

Results: In comparison to control subjects T2D patients had significantly more total Lactobacillus $(+18 \%)$, L. bugaricus $(+13 \%)$, L. rhamnosum $(+37 \%)$ and L. acidophilus $(+48 \%)(P<0.05)$. In contrast, T2D patients had less amounts of total Bifidobacteria $(-7 \%)$ and $B$. adolescentis $(-12 \%)(P<0.05)$. Cluster analysis showed that gut microbiota pattern of T2D patients is characterized by greater numbers of $L$. rhamnosus and $L$. acidophillus, together with lesser numbers of $B$. adolescentis $(P<0.05)$.

Conclusion: The gut microflora in T2D patients is characterized by greater numbers of Lactobacillus and lesser numbers of Bifidobacterium species.

Keywords: microbiota, microflora, metabolic diseases, gut

\section{INTRODUCTION}

The pathophysiology of type 2 diabetes (T2D) involves both environmental and genetic factors. Recently, the gut microflora has emerged as another parameter at the crossroad of these interactions. Several animal and human studies have demonstrated that the gut microflora composition differs between $\mathrm{T} 2 \mathrm{D}$ and controls, and may play a role in the development of insulin resistance and T2Ds (Cani et al., 2008; Delzenne and Cani, 2011). In addition, it has been shown that modification of the gut microbiota by environmental factors may alter body weight and energy metabolism regulation, which may lead to the development of obesity, the major risk factor for T2D (Backhed et al., 2004; Tremaroli et al., 2010).

More than $10^{12}$ microorganisms can be found in the human colon (Eckburg et al., 2005; Andersson et al., 2008). Of these, the Firmicutes, Bacteroides, Actinobacteria, and Proteobacteria phyla are the most prominent (Arumugam et al., 2011). These bacteria can be categorized as commensal, beneficial, or harmful. Some of the deleterious effects include activation of inflammatory processes, alteration of the intestinal barrier, and modification of metabolic pathways (Cani et al., 2008); while beneficial effects include anti-inflammatory effects, improved nutrient digestion, and absorption and regulation of lipid metabolism (Russell et al., 2011). Until now, most studies investigating the gut microbiota composition have used large throughput screening methods to distinguish specific patterns between various patient types (Larsen et al., 2010). One report indicates that Bifidobacterium is underrepresented in T2D patients compared to controls, and may possibly play a role in the development of T2D (Wu et al., 2010b).

Bifidobacterium and Lactobacillus belong to the Actinobacteria and Firmicutes phyla, respectively. In addition to the fact that these 
genera are highly prevalent in the human gut, they also are added as probiotics in foods, making them therefore ideal candidates for potential clinical interventions. Effects of the various strains of bacteria on health can be very divergent, and it still remains unclear which species are responsible for specific metabolic effects. In mice, administration of Lactobacillus casei improves diet-induced obesity and insulin resistance (Naito et al., 2011). However, it has also been shown that presence of certain Lactobacillus species may increase inflammation, which may be related to obesity and T2D (Zeuthen et al., 2006; Santacruz et al., 2009). Increasing the content of gut Bifidobacterium resulting from a prebiotic dietary fiber intervention improved high-fat diet-induced glucose intolerance, insulin secretion, and lowgrade inflammation (Cani et al., 2007). Until now, most studies have been performed in Caucasian populations leaving unknown whether the gut microflora may have similar relationships to metabolism in other ethnicities.

Therefore, the primary aim of this study was to determine if there are specific species differences in total Lactobacillus, L. acidophilus, L. bulgaricus, L. casei, L. rhamnosus, L. plantarum, Total Bifidobacterium, B. longum, B. breve, B. adolescentis, and B. infantis between T2D and control patients in Southern China.

\section{MATERIALS AND METHODS STUDY SUBJECTS}

Diabetic subjects and control subjects were selected for this prospective study based on inclusion criteria listed below between March 2008 and January 2010 at Southern Medical University in Guangzhou, Southern China. Subjects with normal oral glucose tolerance test (OGTT) and HbA1C measurements (see below) were selected as controls. Fifty T2D patients and thirty control subjects of broad age (20-76 years) and body mass index (BMI) $\left(17-36 \mathrm{~kg} / \mathrm{m}^{2}\right)$ ranges were included in this study. Patients were defined as T2D if their fasting and 2-h OGTT glucose values were $\geq 7.0 \mathrm{mmol} / \mathrm{l}$ and $\geq 11.1 \mathrm{mmol} / \mathrm{l}$ respectively, and if they had disease duration of at least 5 years duration but otherwise healthy, age 18-80 years, male or female, stable glycemic control (mean $\mathrm{HbA}_{1 \mathrm{c}}$ levels <8.0\%).

All T2D patients had made lifestyle modifications and 21/50 patients took medications, such as metformin, acarbose, glyburide or Chinese herbal medicine (Pueraria skullcap Coptis soup), and achieved glycemic control $\left(\mathrm{Hb}_{\mathrm{Alc}}\right.$ of 6-7\%, Preprandial blood glucose: $4.0-6.0 \mathrm{mmol} / \mathrm{L}, 2$ - $\mathrm{h}$ postprandial blood glucose: $5.0-8.0 \mathrm{mmol} / \mathrm{L}$.), While the control subjects with normal OGTT had A1C of 4.2-6.3\% (mean, 5.4\%). All patients and control subjects were selected from Southern Medical University hospitals; and most of them lived in the same community and ate a southern Chinese style diet twice a day.

Participants were excluded if there was any evidence of diarrhea, constipation, significant cardiovascular complications, microalbuminuria ( $>40 \mathrm{mg}$ per $24 \mathrm{~h}$ ) or frank proteinuria, evidence of unstable glycemic control (mean $\mathrm{HbA}_{1 \mathrm{c}}$ levels $>8.0 \%$ ), or history of admission for hyperglycemia or hypoglycemia in the 6 months prior to recruitment, other significant renal, hepatic, cardiovascular, or neurological disease; cancer; pregnancy. All participants provided their signed written consent and this study protocol was approved by the Ethical Committee of Southern Medical University.

\section{ANTHROPOMETRY AND METABOLIC QUANTIFICATIONS}

Weight and height were measured to the nearest $0.1 \mathrm{~kg}$ and $0.1 \mathrm{~cm}$, respectively, using a beam medical scale and wall-mounted stadiometer; and BMI was calculated as previously described (Zeuthen et al., 2006). All patients stopped taking their diabetes medications for 3 days and had overnight fast before investigation Glucose tolerance was evaluated using $75 \mathrm{~g}$ oral dose of glucose. Venous blood samples were drawn twice from antecubital vein. The first sample was drawn at 8-10 AM after overnight fasting for at least $12 \mathrm{~h}$, and the second samples was drawn $2 \mathrm{~h}$ after $75 \mathrm{~g}$ oral glucose loading. The first blood sample was examined for baseline metabolic profiles [hemoglobin (HbA1c, C-peptide, C-reactive protein, fasting blood glucose, cholesterol, triglyceride, high-density lipoprotein cholesterol (HDL-C), low-density lipoprotein cholesterol (LDL-C))]. The second blood sample was examined for blood glucose and insulin levels. Fecal specimens were collected within $12 \mathrm{~h}$ after the administration of oral glucose for subsequent measurement of bacterial composition.

\section{Blood analysis}

Blood samples were centrifuged immediately for $10 \mathrm{~min}$ at $2500 \mathrm{RPM}$ at $8-10^{\circ} \mathrm{C}$. Then the serum was removed and frozen at $-70^{\circ} \mathrm{C}$ until assayed. Glucose was assayed in duplicate on a Yellow Springs Instrument 2700 Analyzer (Yellow Springs Instrument, Yellow Springs, $\mathrm{OH}$ ) using the glucose oxidase method, and Glycated hemoglobin (HbAlc) was measured by using a HPLC cation exchange column method (Modular Diabetic Monitoring System; Bio-Rad, Richmond, CA). C-peptide was determined by ELISA (DRG-Diagnostica, Marburg, Germany).

\section{Extraction of bacterial DNA from fecal samples}

Total bacterial DNA was extracted from the fecal samples using DNA stool kit according to the manufacture's protocol (Qiagen, Valencia, CA, USA). DNA concentration and quality in the extracts was determined by agarose gel electrophoresis. Altogether, nine species of probiotic bacteria were chosen for measurement including $L$. acidophilus, L. bulgaricus, L. casei, L. rhamnosus, B. breve, B. longum, and B. infantis. Specific forward and reverse primers for each bacterium were designed (Table 1).

\section{Real-time qPCR}

Bacterial copy numbers in fecal samples from 50 subjects with T2D and 30 controls were quantified by qPCR using the 7500 Fast Real-time PCR system (Applied Biosystems, USA). The qPCR reaction mixture $(20 \mu \mathrm{l})$ was composed of each $0.6 \mu \mathrm{l}$ forward and reverse primer, $6.4 \mu \mathrm{l}$ sterile $\mathrm{ddH}_{2} \mathrm{O}$, ROX Reference Dye (50X) $0.4 \mu \mathrm{l}$, SYBR premix DimerEraser (2X) $10 \mu \mathrm{l}$ (Takara, Biotechnology, Dalian, China) and $2 \mu \mathrm{l}$ fecal DNA as template added in 10 -fold serial dilutions from $10^{2}$ to $10^{12}$ copy/ml. The amplification program consisted of one cycle of $95^{\circ} \mathrm{C}$ for $30 \mathrm{~s}$, followed by 40 cycles of $95^{\circ} \mathrm{C}$ for $5 \mathrm{~s}, 55^{\circ} \mathrm{C}$ for $30 \mathrm{~s}$, and $72^{\circ} \mathrm{C}$ for $34 \mathrm{~s}$. Each fecal sample measurement was performed in duplicate. Standard curves were constructed using 10-fold serial dilutions of fecal bacterial DNA of known concentration. Copy numbers of bacteria in fecal samples were calculated from the threshold cycle values $(\mathrm{Ct})$ and expressed as quantity of bacteria per gram feces. Since data were not normally distributed, these values were subsequently transformed using logarithm. 
Table 1 | PCR primers for detection of Bifidobacterium and Lactobacillus.

\begin{tabular}{lllll}
\hline Gut flora & Forward primer $\mathbf{5}^{\prime} \mathbf{- \mathbf { 3 } ^ { \prime }}$ & Reverse primer $\mathbf{5}^{\prime} \mathbf{- \mathbf { 3 } ^ { \prime }}$ & Size (bp) & References \\
\hline Lactobacillus & AGCAGTAGGGATCTTCCA & CACCGCTACACATGGAG & 341 & Armougom et al., 2009 \\
Lactobacillus acidophilus & GAAAGAGCCAAACCAATGATT & CTTCCCAGATAATTCAACTATCGCTTA & 145 & Wellen and Hotamisligil, 2005 \\
Lactobacillus bulgaricus & GGRTGTTTGTTGACGCTAG & GCCGCCTTTCAAACTTGAATC & 138 & Wu et al., 2010a \\
Lactobacillus casei & GCACCGAGATTCAACATGG & GGTTCTTGGATYTATGCGGTATTAG & 122 & Wu et al., 2010a \\
Lactobacillus rhamnosus & TGCTTGCATCTTGATTTAATTTTG & GTCCATTGTGGAAGATTCCC & 317 & Wu et al., 2010a \\
Lactobacillus plantarum & TGGATCACCTCCTTTCTAAGGAAT & TGTTCTCGGTTTCATTATGAAAAATA & 145 & Haarman and Knol, 2006 \\
Bifidobacterium & GCGTGCTTAACACATGCAAGTC & CACCCGTTTCCAGGAGCTATT & 126 & Armougom et al., 2009 \\
Bifidobacterium longum & GAGACAGAAACTTTCGAGC & GAAGTCTGTGGTATCCAATCC & 112 & Byun et al., 2004 \\
Bifidobacterium breve & TTCCGCATTCGTGTTATTGA & CACATCTTCGCTATCCAGCA & 279 & Byun et al., 2004 \\
Bifidobacterium adolescentis & CTCCAGTTGGATGCATGTC & CGAAGGCTTGCTCCCAGT & 122 & Matsuki et al., 1998 \\
Bifidobacterium infantis & CCATCTCTGGGATCGTCGG & TATCGGGGAGCAAGCGTGA & 563 & Matsuki et al., 1998 \\
\hline
\end{tabular}

\section{STATISTICAL METHODS}

All data are means $\pm S D$. Statistical analyses were performed using STATA 11.0 (Stata Corp, College Station, TX). P-values $<0.05$ was considered statistically significant. Values for bacterial content were transformed using logarithm in order to reach a normal distribution. Unadjusted paired comparisons were done using Student $t$-tests. Adjustments of comparisons for age and gender were performed using analysis of covariance (ANCOVA). Bacterial content patterns were evaluated using cluster analysis of observations, which separates participants into mutually exclusive groups and maximizes differences in the content of a number of bacteria. The cluster k-means procedure in STATA, version 11.0, was used, based on the K-means method. The relationship between the two clusters identified and the presence of diabetes was tested using the chi-squared test. Differences among clusters were investigated using the Kruskal-Wallis analysis of variance test.

\section{RESULTS}

A total of 30 control participants (43\% females) and 50 patients with T2D (54\% females) completed the study. Anthropometric characteristics of the two groups are presented in Table 2. T2D patients were significantly older than the control group $(60 \pm 8$ vs. $41 \pm 11$ years, $P<0.001$ ), and subsequent analyses were thus adjusted for age. T2D patients had significantly greater amount of the total Lactobacillus, as well as greater amounts of L. bulgaricus, L. casei, L. rhamnosus and L. acidophillus, compared to controls (Table 3). However, when adjusted for age and gender, the differences for $L$. case $i$ were no longer significant. T2D patients had less numbers of total Bifidobacterium and B. adolescentis than controls. These differences remained significant when adjusted for age and gender.

Cluster analysis identified two mutually exclusive clusters characterized as follows: cluster 1 had greater numbers of L. rhamnosus and L. acidophillus, and a lesser numbers of B. adolescentis (Figure 1 and Table 4). Cluster 2 had the opposite description, i.e., lesser numbers of L. rhamnosus and L. acidophillus, and a greater numbers of $B$. adolescentis. Interestingly, there was a strong association between the clusters and the presence of diabetes ( $p$-value of chi-squared test: $P<0.001$ ), suggesting that the presence of diabetes may be characterized by a specific
Table 2 | Anthropometric parameters.

\begin{tabular}{lllc}
\hline Parameter & Control $(\boldsymbol{n}=\mathbf{3 0})$ & Diabetic $(\boldsymbol{n}=\mathbf{5 0})$ & $\boldsymbol{P}$-value \\
\hline Sex (M/F) & $17 / 13$ & $23 / 27$ & 0.3 \\
Age (years) & $41 \pm 11$ & $60 \pm 8$ & $<0.001$ \\
Weight $(\mathrm{kg})$ & $64.2 \pm 9.0$ & $62.9 \pm 10.0$ & 0.7 \\
BMl $\left(\mathrm{kg} / \mathrm{m}^{2}\right)$ & $23.9 \pm 3.0$ & $24.7 \pm 3.8$ & 0.5 \\
\hline
\end{tabular}

gut microflora pattern. Thus, greater numbers of L. rhamnosus and $L$. acidophillus, and a lesser numbers of B. adolescentis, are high specificity descriptors of Cluster 1 for T2D. Sensitivity and specificity calculations of this cluster analysis showed that for such bacterial analysis and prediction of T2D, the sensitivity $=0.62$, specificity $=0.97$, positive predictive value $=0.97$ and negative predictive value $=0.60$.

Patients took multiple medications including metformin, the prescriptions were personalized so that there were different doses of medications in variety combinations. We compared metformin group with other patients group, no difference has been observed.

\section{DISCUSSION}

Previous studies have shown that specific gut microbiota composition is linked to the presence of T2D (Larsen et al., 2010; Wu et al., 2010b). In the present study, we showed that in a Southern Chinese population, T2D patients have increased numbers of L. Bulgaricum, L. rhamnosus and L. acidophillus and decreased numbers of B. Adolescentis, compared to controls. Together, this set of bacteria formed a cluster characteristic of T2D patients.

Previous studies have shown that T2D patients had greater numbers of Lactobacillus and lesser numbers of Bifidobacterium in their gut as measured in feces compared to non-diabetic patients. Our results obtained in Chinese subjects are in line with these studies. In addition, we further measured individual species of Lactobacillus and Bifidobaterium and showed that several Lactobacillus species, namely L. bulgaricum, L. rhamnosus and L. acidophillus are increased in number in T2D patients. The role of Lactobacillus in metabolic diseases remains unclear. Administration of $L$. casei strain improved glucose tolerance in diet-induced obese mice (Naito et al., 2011). In contrast, other studies point to a pro-inflammatory role of Lactobacillus. 
Table 3 | Comparisons of bacteria amounts between controls and diabetic patients (bacterial values transformed using logarithm).

\begin{tabular}{lcccc}
\hline & Control $(\boldsymbol{n}=\mathbf{3 0})$ & Diabetic $(\boldsymbol{n}=\mathbf{5 0})$ & Unadjusted $\boldsymbol{P}$-value & $P$-value adjusted for age and gender \\
\hline Lactobacillus & & & & 0.03 \\
Lactobacillus bulgaricus & $4.6 \pm 0.8$ & $5.2 \pm 0.6$ & 0.0006 & 0.19 \\
Lactobacillus casei & $4.5 \pm 0.9$ & $5.0 \pm 1.0$ & 0.03 & 0.68 \\
Lactobacillus plantarum & $4.6 \pm 0.7$ & $4.7 \pm 0.9$ & 0.9 & 0.004 \\
Lactobacillus rhamnosus & $2.7 \pm 0.4$ & $3.7 \pm 1.0$ & $<0.0001$ & 0.01 \\
Lactobacillus acidophillus & $2.9 \pm 0.6$ & $4.3 \pm 1.4$ & $<0.0001$ & 0.0005 \\
Lactobacillus (total) & $6.6 \pm 0.5$ & $7.8 \pm 0.9$ & $<0.0001$ & 0.2 \\
Bifidobacterium & & & & 0.4 \\
Bifidobacterium longum & $3.9 \pm 0.8$ & $3.7 \pm 0.7$ & 0.15 & 0.0003 \\
Bifidobacterium breve & $3.9 \pm 0.3$ & $3.9 \pm 0.3$ & 0.4 & 0.3 \\
Bifidobacterium adolescentis & $4.0 \pm 0.3$ & $3.5 \pm 0.4$ & $<0.0001$ & 0.002 \\
Bifidobacterium infantis & $3.9 \pm 1.0$ & $4.0 \pm 0.9$ & 0.7 & $<.0001$
\end{tabular}

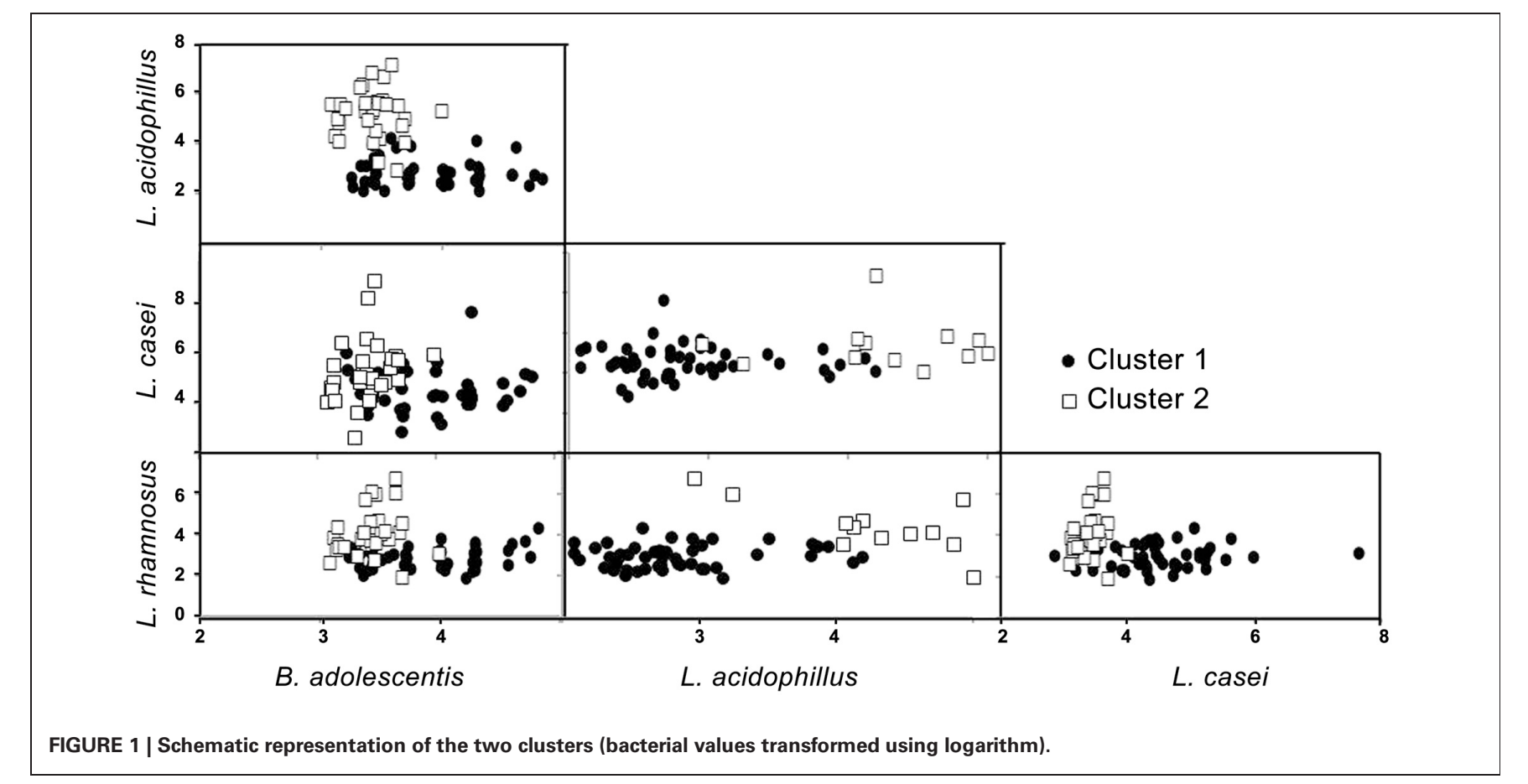

For example, obese individuals have greater numbers of Lactobacillus in their feces, compared to lean controls (Armougom et al., 2009). Obesity is characterized by a chronic, low-grade inflammation, which may lead to the development of insulin resistance and ectopic fat deposition (Wellen and Hotamisligil, 2005). Although the mechanisms remain unknown, it has been speculated that modifications of the gut microbiota may increase local and plasma lipopolysaccharide (LPS), which may in turn lead to inflammation, hepatic fat accumulation, insulin resistance, and T2D (Cani et al., 2008). In our study, Lactobacillus levels were not related to plasma C-reactive proteins concentrations, a measure of inflammation (data not shown). However, presence of such bacteria may locally induce inflammation or other cytokines that we did not measure, such as TNF- $\alpha$ or IL-6.
We also showed that Bifidobacterium numbers were decreased in T2D patients. Our results support previous studies, showing that both insulin resistant and obese patients have decreased Bifidobacterium numbers in their gut (Larsen et al., 2010; Wu et al., 2010b). In the present study, the only species that was significantly different was $B$. adolescentis. $B$. adolescentis has been shown to reduce intestinal permeability, thus providing a better protection against endotoxin translocation (Wu et al., 2010a). Previous studies suggest that high-fat diet-induced endotoxemia could modify gut microbiota, and lead to a decrease in Bifidobacterium species (Cani et al., 2007). The pathogenesis of T2D is strongly influenced by environmental factors, such as diet. Therefore, it remains possible that poor nutritional habits may in the long term contribute to modification of the gut microbiota, which may in turn play a role in the development 
Table 4 | Comparisons of bacteria amount by clusters (bacterial values transformed using logarithm).

\begin{tabular}{lcccc}
\hline & Cluster $\mathbf{1}(\boldsymbol{n}=\mathbf{3 2})$ & Cluster $\mathbf{2}(\boldsymbol{n}=\mathbf{4 8})$ & Unadjusted $\boldsymbol{P}$-value & $\boldsymbol{P}$-value adjusted for age and gender \\
\hline Cases of Diabetes (\%) & $31(97 \%)$ & $19(40)$ & $<0.0001$ & \\
Lactobacillus & & & & 0.09 \\
Lactobacillus bulgaricus & $5.1 \pm 0.6$ & $4.9 \pm 0.8$ & 0.003 & 0.2 \\
Lactobacillus casei & $5.2 \pm 1.2$ & $4.5 \pm 0.8$ & 0.21 & 0.6 \\
Lactobacillus plantarum & $4.8 \pm 0.9$ & $4.6 \pm 0.8$ & $<0.0001$ & 0.0001 \\
Lactobacillus rhamnosus & $4.0 \pm 1.0$ & $2.9 \pm 0.5$ & $<0.0001$ & 0.0001 \\
Lactobacillus acidophillus & $5.1 \pm 0.9$ & $2.8 \pm 0.5$ & 0.001 & 0.6 \\
Lactobacillus (total) & $7.7 \pm 0.9$ & $7.0 \pm 0.9$ & & 0.2 \\
Bifidobacterium & & & 0.06 & 0.003 \\
Bifidobacterium longum & $3.5 \pm 0.7$ & $3.9 \pm 0.8$ & 0.4 & 0.7 \\
Bifidobacterium breve & $3.9 \pm 0.3$ & $3.9 \pm 0.3$ & $<0.0001$ & 0.4 \\
Bifidobacterium adolescentis & $3.4 \pm 0.2$ & $3.9 \pm 0.4$ & 0.5 & 0.03 \\
Bifidobacterium infantis & $3.9 \pm 0.8$ & $4.0 \pm 1.0$ & & 0.03 \\
Bifidobacterium (total) & $10.7 \pm 0.7$ & $11.2 \pm 0.9$ & & \\
\hline
\end{tabular}

of T2D. Further mechanistic studies will be required to assess causal relationships.

Based on these various differences in gut microbiota composition between T2D patients and the control individuals, we further aimed to assess whether T2D could be characterized by a specific "bacterial signature." Using cluster analysis, we identified two mutually exclusive clusters. Cluster 1 was characterized by high amount of $L$. Rhamnosus and L. acidophillus, while lower amount of B. adolescentis. Interestingly, all except one individual in Cluster 1 had T2D, suggesting that such analysis can provide high specificity for the disease. Indeed, the specificity for such test was 0.97 , and the positive predictive value reached the same score of 0.97 . This suggests that most individuals identified with Cluster 1 have a high probability of being diabetic. However, the number of cases of "false negative" remained high, with a sensitivity of 0.62 and a negative predictive value of 0.60 . Of note, it is not clear why one individual in Cluster 1 did not have diabetes. However, considering the very high sensitivity of Cluster in patients with diabetes, it is conceivable that this one patient represents the metabolic phenotype of diabetes without the usual clinical measures of diabetes. On the other hand, the high false negative rate of Cluster 1 designation for diabetes patients suggests that Cluster 1 designation represents a unique metabolic subset of patients with diabetes. These suggestions may lead to further investigations to understand the mechanisms of these differences in the patient groups. Therefore, more accurate evaluation and refined analysis of gut bacteria will need to be performed. In addition, the relationship between the changes of the gut

\section{REFERENCES}

Andersson, A. F., Lindberg, M., Jakobsson, H., Backhed, F., Nyren, P., and Engstrand, L. (2008). Comparative analysis of human gut microbiota by barcoded pyrosequencing. PLOS ONE 3:e2836. doi: 10.1371/journal.pone.0002836

Armougom, F., Henry, M., Vialettes,

B., Raccah, D., and Raoult,
D. (2009). Monitoring bacterial community of human gut microbiota reveals an increase in Lactobacillus in obese patients and Methanogens in anorexic patients. PLOS ONE 4:e7125. doi: 10.1371/journal.pone.0007125

Arumugam, M., Raes, J., Pelletier, E., Le, P. D., Yamada, T., Mende, D. R., et al. (2011). Enterotypes of

microbiota and the development of T2Ds should be investigated in a prospective cohort study, which would be useful to identify individuals at high risk for T2D. Although these preliminary results need to be further validated by larger number of patients and more refined bacterial analysis, they may provide some direction for development of future diagnostic tool to identify individuals at high risk for T2D and initiate early prevention and treatment.

The potential clinical impacts of this study are 2 -fold: first, we have identified a bacterial signature of T2D, which may be further developed and possibly used as a future diagnostic tool for identification of patients at risk for T2D. Second, our findings may lead to dietary recommendations for gut microbiota modulation such as diets promoting increased Bifidobacterium. In conclusion, we showed that T2D patients can be characterized by a specific fecal bacterial pattern of Lactobacillus and Bifidobacterium species. Modification of these bacteria levels, may influence T2D.

\section{ACKNOWLEDGMENTS}

This research was partially supported by the Major State Basic Research Development Program of China 973 Program (No. 2011CB504006), Songshan Lake Sci. \& Tech. Industry Park Fund (No. 2010B025 \& No. 2010B026), Ph.D. Programs Foundation of Ministry of Education of China (No. 20104433110014), Guangdong science and Technology Research Fund (No. 2010B090400041) and Guangdong Department of Education Fund. The United State Department of Veterans Affairs and Hirshberg Foundation.

the human gut microbiome. Nature 473, 174-180.

Backhed, F., Ding, H., Wang, T., Hooper, L. V., Koh, G. Y., Nagy, A., et al. (2004). The gut microbiota as an environmental factor that regulates fat storage. Proc. Natl. Acad. Sci. U.S.A. 101, 15718-15723.

Byun, R., Nadkarni, M. A., Chhour, K. L., Martin, F. E., Jacques, N. A., and Hunter, N. (2004). Quantitative analysis of diverse Lactobacillus species present in advanced dental caries. J. Clin. Microbiol. 42, 3128-3136.

Cani, P. D., Bibiloni, R., Knauf, C., Waget, A., Neyrinck, A. M., Delzenne, N. M., et al. (2008). Changes in gut microbiota control metabolic endotoxemia-induced 
inflammation in high-fat dietinduced obesity and diabetes in mice. Diabetes 57, 1470-1481.

Cani, P. D., Neyrinck, A. M., Fava, F., Knauf, C., Burcelin, R. G., Tuohy, K. M., et al. (2007). Selective increases of bifidobacteria in gut microflora improve high-fat-diet-induced diabetes in mice through a mechanism associated with endotoxaemia. Diabetologia 50, 2374-2383.

Delzenne, N. M., and Cani, P. D. (2011). Gut microbiota and the pathogenesis of insulin resistance. Curr. Diab. Rep. 11, 154-159.

Eckburg, P. B., Bik, E. M., Bernstein, C. N., Purdom, E., Dethlefsen, L., Sargent, M., et al. (2005). Diversity of the human intestinal microbial flora. Science 308, 1635-1638.

Haarman, M., and Knol, J. (2006). Quantitative real-time PCR analysis of fecal Lactobacillus species in infants receiving a prebiotic infant formula. Appl. Environ. Microbiol. 72, 2359-2365.

Larsen, N., Vogensen, F. K., van den Berg, F. W., Nielsen, D. S., Andreasen, A. S., Pedersen, B. K., et al. (2010). Gut microbiota in human adults with type 2 diabetes differs from non-diabetic adults. PLoS ONE 5:e9085. doi: 10.1371/journal.pone.0009085

Matsuki, T., Watanabe, K., Tanaka, R., and Oyaizu, H. (1998). Rapid identification of human intestinal bifidobacteria by $16 \mathrm{~S}$ rRNA-targeted species- and group-specific primers. FEMS Microbiol. Lett. 167, 113-121.

Naito, E., Yoshida, Y., Makino, K., Kounoshi, Y., Kunihiro, S., Takahashi, R., et al. (2011). Beneficial effect of oral administration of Lactobacillus casei strain Shirota on insulin resistance in diet-induced obesity mice. J. Appl. Microbiol. 110, 650-657.

Russell, D. A., Ross, R. P., Fitzgerald, G. F., and Stanton, C. (2011). Metabolic activities and probiotic potential of bifidobacteria. Int. J. Food Microbiol. 149, 88-105.

Santacruz, A., Marcos, A., Warnberg, J., Marti, A., Martin-Matillas, M., Campoy, C., et al. (2009). Interplay between weight loss and gut microbiota composition in overweight adolescents. Obesity (Silver Spring) 17, 1906-1915.

Tremaroli, V., Kovatcheva-Datchary, P., and Backhed, F. (2010). A role for the gut microbiota in energy harvesting? Gut 59, 1589-1590.
Wellen, K. E., and Hotamisligil, G. S. (2005). Inflammation, stress, and diabetes. J. Clin. Invest. 115, 1111-1119.

Wu, J., Wang, X., Cai, W., Hong, L., and Tang, Q. (2010a). Bifidobacterium adolescentis supplementation ameliorates parenteral nutrition-induced liver injury in infant rabbits. Dig. Dis. Sci. 55, 2814-2820.

Wu, X., Ma, C., Han, L., Nawaz, M. Gao, F., Zhang, X., et al. (2010b). Molecular characterisation of the faecal microbiota in patients with type II diabetes. Curr. Microbiol. 61, 69-78.

Zeuthen, L. H., Christensen, H. R., and Frokiaer, H. (2006). Lactic acid bacteria inducing a weak interleukin-12 and tumor necrosis factor alpha response in human dendritic cells inhibit strongly stimulating lactic acid bacteria but act synergistically with gram-negative bacteria. Clin. Vaccine Immunol. 13, 365-375.

Conflict of Interest Statement: KimAnne Lê is employed by Nestec Ltd. which is a subsidiary of Nestlé Ltd. and provides professional assistance, research, and consulting services for food, dietary, dietetic, and pharmaceutical products of interest to Nestlé Ltd. The other authors declare that the research was conducted in the absence of any commercial or financial relationships that could be construed as a potential conflict of interest.

Received: 16 December 2011; accepted: 28 December 2012; published online: 31 January 2013.

Citation: Lê K-A, Li Y, Xu X, Yang W, Liu T, Zhao X, Tang YG, Cai D, Go VLW, Pandol S and Hui H (2013) Alterations in fecal Lactobacillus and Bifidobacterium species in type 2 diabetic patients in Southern China population. Front. Physio. 3:496. doi: 10.3389/fphys. 2012.00496

This article was submitted to Frontiers in Gastrointestinal Sciences, a specialty of Frontiers in Physiology.

Copyright (c) $2013 \mathrm{Le}, \mathrm{Li}, \mathrm{Xu}$, Yang, Liu, Zhao, Tang, Cai, Go, Pandol and Hui. This is an open-access article distributed under the terms of the Creative Commons Attribution License, which permits use, distribution and reproduction in other forums, provided the original authors and source are credited and subject to any copyright notices concerning any third-party graphics etc. 